\title{
A Novel Photosynthesis of Carboxymethyl Starch-Stabilized Silver Nanoparticles
}

\author{
M. A. El-Sheikh \\ National Research Centre, Textile Research Division, El-Behouth Street, Dokki, P.O. Box 12311, Giza, Egypt \\ Correspondence should be addressed to M. A. El-Sheikh; dr.manal.elsheikh@gmail.com
}

Received 13 August 2013; Accepted 4 November 2013; Published 29 January 2014

Academic Editors: A. Jacquot, Y.-L. Kuo, Z. Peng, R.-C. Sun, A. Tonkikh, and Z. Zhou

Copyright (C) 2014 M. A. El-Sheikh. This is an open access article distributed under the Creative Commons Attribution License, which permits unrestricted use, distribution, and reproduction in any medium, provided the original work is properly cited.

\begin{abstract}
The water soluble photoinitiator (PI) 4-(trimethyl ammonium methyl) benzophenone chloride is used for the first time in the synthesis of silver nanoparticles (AgNPs). A new green synthesis method involves using PI/UV system, carboxymethyl starch (CMS), silver nitrate, and water. A mechanism of the reduction of silver ions to AgNPs by PI/UV system as well as by the newly born aldehydic groups was proposed. The synthesis process was assessed by UV-vis spectra and TEM of AgNPs colloidal solution. The highest absorbance was obtained using CMS, PI and $\mathrm{AgNO}_{3}$ concentrations of $10 \mathrm{~g} / \mathrm{L}, 1 \mathrm{~g} / \mathrm{L}$, and $1 \mathrm{~g} / \mathrm{L}$, respectively; $40^{\circ} \mathrm{C} ; 60 \mathrm{~min}$; $\mathrm{pH} 7$; and a material: liquor ratio 1:20. AgNPs so-obtained were stable in aqueous solution over a period of three weeks at room temperature $\left(\sim 25^{\circ} \mathrm{C}\right)$ and have round shape morphology. The sizes of synthesized AgNPs were in the range of 1-21 nm and the highest counts $\%$ of these particles were for particles of 6-10 and 1-3 nm, respectively.
\end{abstract}

\section{Introduction}

Silver nanoparticles have unique optical, electrical, and biological properties that have attracted significant attention due to their potential use in many applications, such as catalysis, biosensing, drug delivery, and medical textiles [1-5].

Techniques used in the formation of nanosized metal particles are Top-down and Bottom-up techniques. Top-down is mechanically based on diminishing processes that start from bulk metals. Bottom-up technique predominantly employs the chemical or electrochemical reduction of metal salts in solution. Thermolytic, photolytic, radiolytic, laser irradiation, microwave heating, or sonochemical procedures, as well as the decomposition of appropriate metastable precursor molecules, complete the wide spectrum of synthetic pathways to metal nanoparticles. The formation of nanoparticles occurs when individual, neutral atoms are subjected to collide with other atoms, resulting in a nucleation to smaller or larger particles [6].

Sodium borohydride, hydroxylamine hydrochloride, dimethylamine borane, sodium citrate, hydrazine monohydrate, sodium formate, trimethylamine borane, sodium trimethoxyborohydride, and formaldehyde have all become routine reducing agents for the generation of metal NPs.
Among the various reducing agents, $\mathrm{BH}^{4-}$ plays the dominant role. These chemicals are highly reactive and cause potential environmental and biological risks $[7,8]$. Utilization of nontoxic chemicals, environmentally gentle solvents and renewable materials is one of the key issues that worth important consideration in a green synthesis policy.

Direct photoreduction and photosensitization are powerful approaches for the in situ synthesis in polymer matrixes [9-16]. The heart of the photochemical approach is the generation of $\mathrm{M}^{0}$ (metal nanoparticles) in such conditions that their precipitation is thwarted. Metal nanoparticles can be formed through direct photoreduction of a silver source, silver salt, or complex, or reduction of silver ions using photochemically generated intermediates, such as radicals. The photoreduction is often promoted by dyes dispersed or dissolved in the polymer or present in the chemical structure of the matrix. The visible light photoinitiator "camphorquinone" was used to generate electron-donating radicals upon photolysis. Subsequent oxidation of these radicals to the corresponding cations in the presence of silver hexafluoroantimonate $\left(\mathrm{AgSbF}_{6}\right)$ leads to the simultaneous formation of silver nanoparticles. The key step of the process is the reaction of silver cations with photogenerated transient species that are able to reduce them to silver metal atoms. Two classes of 
photoinduced reactions were used to produce these primary radicals. The first one is based on the reaction of an electron rich molecule (e.g., amine, thiol, and ether) with the highly oxidant triplet state of a sensitizer excited upon absorption of the actinic photons. The second one involves the direct hemolytic photocleavage of a sigma bond (mainly C-C bonds adjacent to a carbonyl) [17].

Since the polymers prevent agglomeration and precipitation of the particles, they have been frequently employed as capping or stabilizing agents in the chemical synthesis of metal nanoparticles. Among methods of green synthesis of silver nanoparticles, water is used as an environmentally benign solvent and polysaccharides as a capping agent, or in some cases polysaccharides serve as both a reducing and a capping agent $[4,8,18-27]$. In a case of dual polysaccharide function, silver nanoparticles were synthesized by the reduction of $\mathrm{Ag}^{+}$inside of nanoscopic starch templates. The extensive network of hydrogen bonds in the templates provides surface passivation or protection against nanoparticle aggregation [26]. Biosynthesis is also an alternative green technique for synthesis of silver nanoparticles [2, 28-31].

The so-called "alcohol reduction process" is a very general process for the production of metal nanoparticles, often stabilized by organic polymers. In general, the alcohols which were useful reducing agents contained $\alpha$-hydrogen and were oxidized to the corresponding carbonyl compounds. The oxidation of primary alcohols $\left(\mathrm{R}-\mathrm{CH}_{2} \mathrm{OH}\right)$ by $\mathrm{Ag}^{+}$is also well established; the reaction is slow and requires heating to be accelerated as follows:

$$
2 \mathrm{Ag}^{+}+\mathrm{R}-\mathrm{CH}_{2} \mathrm{OH} \longrightarrow 2 \mathrm{Ag}^{0}+2 \mathrm{H}^{+}+\mathrm{R}-\mathrm{CHO}
$$

However, such a reaction is a very slow process and constitutes a minor route for $\mathrm{Ag}^{+}$reduction in comparison with the photoinduced generation of AgNPs described hereafter $[11,32]$.

Bottom et al. [33, 34] developed and assessed the efficiency of a novel, water-soluble photosensitizer, 4-(trimethyl ammonium methyl) benzophenone chloride, and used it for the photoinitiated graft copolymerization of 2-hydroxyethyl acrylate onto cellulose. El-Sheikh and coworkers [35-38] further utilized this efficient photoinitiator in the graft copolymerization of acrylic acid and acrylamide onto carboxymethyl starch and in the photooxidation of starch. The above cited references are the only ones found in the literature about the using of 4-(trimethyla mmonium methyl) benzophenone chloride. To the best of the author's knowledge and according to literature survey, the later has never been used in the photosynthesis of AgNPs.

Benzophenone compounds are powders. They can absorb and dissipate UV radiation. Benzophenone compounds are used in bath products, makeup products, hair products, sunscreens, and skin care products. They protect the skin from the harmful effects of the sun.

According to the literature survey, one can describe the process as a "novel" process. According to the use of the benzophenone compounds in cosmetics, one can describe the photoinitiator used in this research work as a safe, ecofriendly, and green compound.
In this work, a novel green method was adopted to synthesize AgNPs using the water soluble 4-(trimethyl ammonium methyl) benzophenone chloride/UV system and silver nitrate as a precursor in the presence of a water soluble polymer (CMS). While PI photogenerate AgNPs, CMS act as both a reducing and stabilizing agent. All components are water soluble, the process is "simple and easy," and the synthesis conditions used are mild. Parameters affecting the synthesis of AgNPs such as PI, silver nitrate, and CMS concentrations as well as reaction time and temperature are studied. The synthesized AgNPs were assessed by measuring the absorbance of the colloidal solution, the size, the shape, and the stability of the synthesized AgNPs.

\section{Experimental}

2.1. Materials. Native maize starch (St.) was kindly supplied by the Egyptian Company for Starch and Glucose Manufacture, Cairo, Egypt.

4-(Trimethyl ammonium methyl) benzophenone chloride, a water soluble photoinitiator that belongs to the benzophenone series (UV Absorbers), was supplied by "The associated Octel Ltd., Widnes, UK," and used without further purification.

Monochloroacetic acid, sodium hydroxide, sodium carbonate, silver nitrate, hydrochloric acid, acetic acid, ethanol, and isopropanol were laboratory grade chemicals.

2.2. Equipment. The irradiation reaction vessel consists of a quick fit water-cooled $125 \mathrm{~W}$ medium-pressure $\mathrm{Hg}$ lamp assembly as a UV irradiation source immersed in a quick fit $150 \mathrm{~mL}$ cylindrical tube. The total dose of the UV irradiation was controlled by controlling the time of exposure, that is, the reaction time. The reaction temperature was controlled using a thermostatic water bath.

\subsection{Method}

2.3.1. Carboxymethylation. Water soluble carboxymethyl starch with DS $=0.2$ was prepared according to a reported method [39]. In this method, $100 \mathrm{~g}$ of maize starch was placed in a sealable bottle and mixed together with a known volume of isopropanol. An aqueous solution of sodium hydroxide ( $0.5 \mathrm{~mole} / \mathrm{mole}$ St.) was added dropwise to the starchisopropanol mixture under stirring until the whole amount of sodium hydroxide was added. The sodium salt solution of monochloroacetic acid ( $0.2 \mathrm{~mole} / \mathrm{mole}$ St. $)$, prepared by the reaction of monochloroacetic acid with the equivalent amount of sodium carbonate, was added dropwise to the starch-isopropanol-sodium hydroxide mixture under continuous stirring until the complete addition of the sodium monochloroacetate solution. Stirring was then stopped and the bottle was closed and kept at $30^{\circ} \mathrm{C}$ for 24 hours. After carboxymethylation, the CMS samples were washed with ethanol:water solution $(80: 20)$ while excess alkali was neutralized using acetic acid. After washing, the CMS samples were filtered and oven-dried at $70^{\circ} \mathrm{C}$. 
2.3.2. Preparation of Silver Nanoparticles. In a beaker, a known weight of CMS was mixed with certain volume of distilled water using a mechanical stirrer. After complete dissolution of CMS, an aqueous solution of PI was added to the CMS solution followed by adding silver nitrate solution under continuous stirring until complete mixing of the whole contents. Doing so, the beaker contents were poured in the irradiation tube and transferred to a thermostatic water bath with a magnetic stirrer. The UV lamp is now immersed in the solution to just above the bottom of the tube to allow the magnet to move and to allow the whole solution to be exposed to the UV irradiation. The temperature was then allowed to rise gradually until the required temperature is reached. Finally, the UV lamp is switched on and the whole contents were kept at the synthesis temperature for a known period of time under continuous stirring. After synthesis, the yellowish brown colloidal solution is kept in a sealable bottle at room temperature $\left(25^{\circ} \mathrm{C}\right)$.

2.4. Characterizations and Analyses. The DS of the carboxymethylated starch samples was determined via the determination of the carboxyl content according to a reported method [40].

The UV-vis spectra of silver nanoparticles and PI were recorded using UV-2401, UV-vis Spectrophotometer, Shimadzu, Japan, at wavelength range from 190 to $550 \mathrm{~nm}$. $\lambda$ max at $256 \mathrm{~nm}$ is characteristic of PI, whereas $\lambda$ max at $390-420$ is characteristic of silver nanoparticles. Synthesis of silver nanoparticles is expressed as the absorbance of the colloidal solution of the samples under test. The absorbance, the broadening, and the wavelength of the band measure the intensity of the colloidal solution, that is, the conversion of silver ions to AgNPs. Very concentrated AgNPs samples did not show one smooth band (either sharp or broad) but showed a number of crowded sharp bands. This behavior leads to false readings. So, concentrated samples which showed this behavior were diluted " $x$ " times and the obtained absorbance value was then multiplied by " $x$ " to obtain the actual absorbance value. The accuracy of this dilution technique was tested by comparing the absorbance readings of certain samples with moderate concentration before and after dilution. The readings were found approximately the same after multiplying by " $x$ " times of dilutions.

Transmission Electron Microscope was used to characterize AgNPs. Thus, the shape and size of the synthesized silver nanoparticles were characterized by means of a JEOLJEM-1200 Transmission Electron Microscope. The samples were prepared by placing a drop of the colloidal solution on a 400-mesh copper grid coated by an amorphous carbon film and evaporating the solvent in air at room temperature. The average diameter of the silver nanoparticles was determined from the diameter of nanoparticles found in several chosen areas in enlarged microphotographs. The particle size was measured from the TEM image using the software "Revolution v1.6.0b195," a simple electron microscope tool for acquiring images, maps, and spectra using the Spectral Engine, 1999-2002 4pi analysis, Inc.

Stability of the silver nanoparticles was tested by storing samples in sealed glass bottles in a dark place at room temperature $\left(\sim 25^{\circ} \mathrm{C}\right)$ and then recording the spectra of the colloidal solutions after one week, two weeks, and three weeks of storing using UV-vis Spectrophotometer.

\section{Results and Discussions}

3.1. Mechanism of Photosynthesis of AgNPs. 4-(Trimethyl ammonium methyl) benzophenone chloride structure is represented in Scheme 1(a). Under UV light excitation, 4(trimethyl ammonium methyl) benzophenone chloride (in an aqueous medium) is first promoted to its excited singlet state (1); then, via fast intersystem crossing (ISC), it converts into triplet state (2). In the presence of CMS, which acts as a $\mathrm{H}$-donor, the triplet transient state can undergo hydrogen abstraction with CMS to yield reactive radical species (3). Inactive species formation could take place by the combination of two radicals of PI (4) forming the pinnacol derivative (I) (Scheme 1(b)). The greater the extent of formation of compound I is, the more pronounced the inactivation of the photosynthesis process is [35-37]. The formation of CMS radical (3) initiates a photooxidation reaction which leads to the generation of new aldehydic end groups in the CMS chain. The hydroxyl and carboxyl groups originally present in the CMS molecules in addition to the newly generated aldehydic end groups, due to the photooxidation reaction, all can act as reducing groups of the $\mathrm{Ag}^{+}$to $\mathrm{Ag}^{0}((6)$ and (7)). In addition, the radical of the PI formed according to (3) can further reduce the silver ions to AgNPs as shown in (5). Accordingly, one can expect a very efficient reducing system that arose from (a) reducing groups of CMS, molecules, (b) radicals of CMS and (c) radicals of PI. At the same time while the reduction is keeping on and AgNPs grow gradually, CMS will further form a stable protection layer on the AgNPs surface.

Factors affecting the reduction and stability as well as the shape and size of the formed AgNPs along with reaction mechanism are given below.

\subsection{Effect of Photoinitiator Concentration on the Formation} of Silver Nanoparticles. Different concentrations of PI (from 0.5 to $2 \mathrm{~g} / \mathrm{L}$ ) were used to study the effect of increasing the concentration of PI on the extent of photosynthesis of AgNPs. Thus, photosynthesis was carried out using PI/UV system for one hour of UV irradiation at $30^{\circ} \mathrm{C}$ using CMS and $\mathrm{AgNO}_{3}$ concentrations of $50 \mathrm{~g} / \mathrm{L}$ and $1 \mathrm{~g} / \mathrm{L}$, respectively, and a material: liquor ratio ( $\mathrm{M}: \mathrm{L}$ ratio) $1: 20$ at $\mathrm{pH}$. The dependence of the absorbance of the colloidal solution of the synthesized AgNPs on the concentration of PI is shown in Figure 1. Figure 1 shows that, regardless of the concentration of PI, four intensive bands with symmetrical bell shapes and high absorbance values at $390-420 \mathrm{~nm}$ are formed. This indicates the formation of AgNPs [26,41] and reflects the efficiency of the current system in synthesizing AgNPs at the synthesis conditions. The results obtained from Figure 1 shows also that increasing the concentration of PI from 0.5 to $1 \mathrm{~g} / \mathrm{L}$ is accompanied by a dramatic increase in the absorbance of the colloidal solution. Maximum absorbencies (at $\lambda=$ $410 \mathrm{~nm}$ ) of the colloidal solutions of AgNPs synthesized using $0.5 \mathrm{~g} / \mathrm{L}$ and $1 \mathrm{~g} / \mathrm{L}$ were 40.9 and 55.8 , respectively. Incorporation of PI into the PI/UV system enhances the formation 


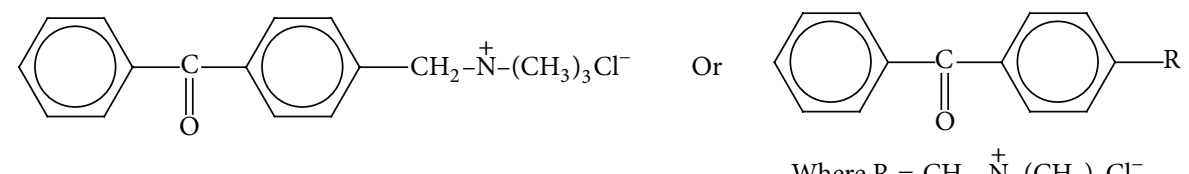

(a)

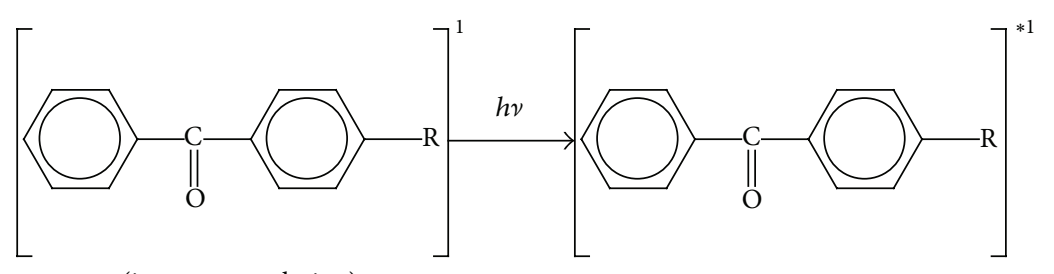

(in aqueous solution)<smiles>[R]c1ccc(C(=O)c2ccc(C)c(I)c2)cc1</smiles>

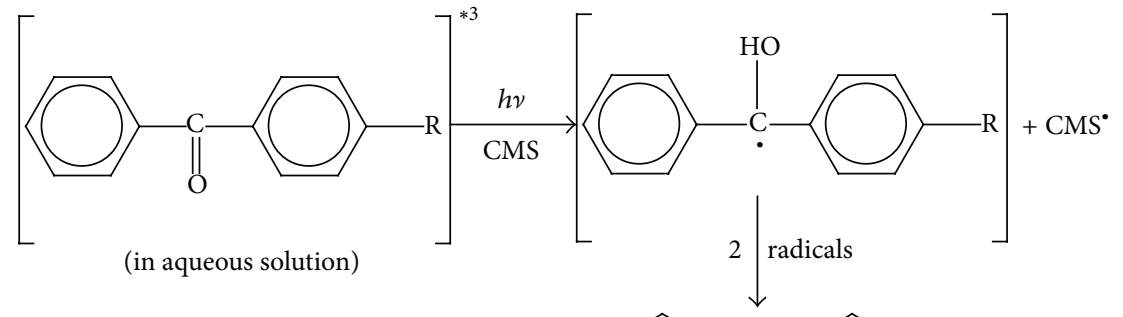<smiles>[R]c1ccc(C(O)(c2ccccc2)c2ccc([R])cc2)cc1</smiles>

$(4)$

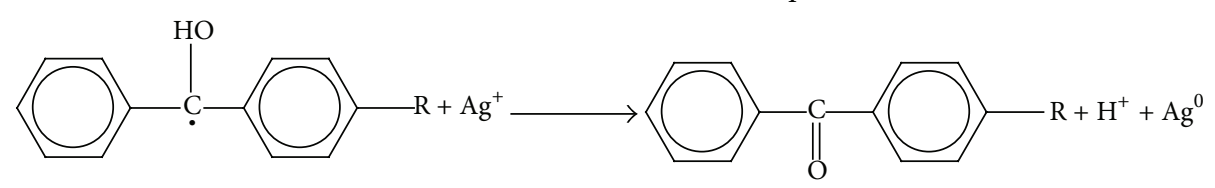

$$
\begin{aligned}
& 2 \mathrm{Ag}^{+}+\mathrm{R}^{\prime}-\mathrm{CH}_{2} \mathrm{OH} \longrightarrow 2 \mathrm{Ag}^{0}+2 \mathrm{H}^{+}+\mathrm{R}^{\prime}-\mathrm{CHO} \\
& 2 \mathrm{Ag}^{+}+\mathrm{R}^{\prime}-\mathrm{CHO}+\mathrm{H}_{2} \mathrm{O} \longrightarrow 2 \mathrm{Ag}^{0}+2 \mathrm{H}^{+}+\mathrm{R}^{\prime}-\mathrm{COOH}
\end{aligned}
$$

(b)

Scheme 1: (a) Chemical structure of PI. (b) Mechanism of photosynthesis of AgNPs. 


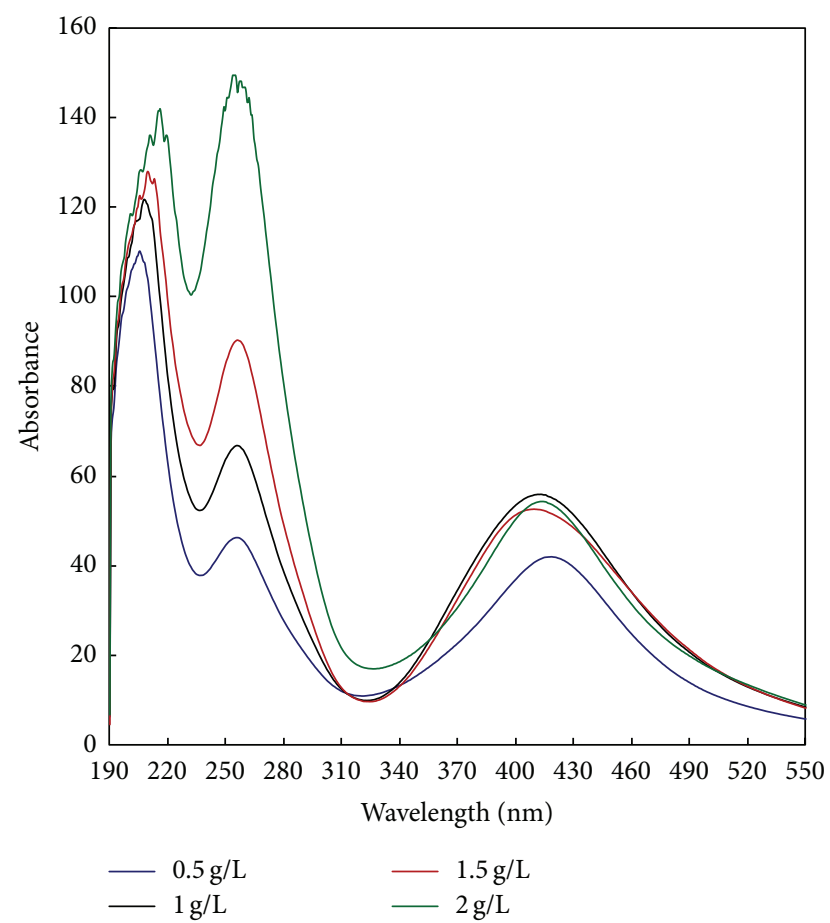

FIGURE 1: Effect of photoinitiator concentration on the synthesis of silver nanoparticles [CMS], $50 \mathrm{~g} / \mathrm{L}$; [PI], 0.5-2 g/L; $\left[\mathrm{AgNO}_{3}\right], 1 \mathrm{~g} / \mathrm{L}$; temp., $30^{\circ} \mathrm{C}$; time, $60 \mathrm{~min}$.; $\mathrm{M}: \mathrm{L}$ ratio, $1: 20 ; \mathrm{pH}, 7$.

of $\mathrm{CMS}^{*}$ radical (3). Consequently, initiates a photooxidation reaction resulting in the generation of new aldehydic end groups in the CMS chain. In their turn, the newly born aldehydic groups reduce the $\mathrm{Ag}^{+}$to $\mathrm{Ag}^{0}$. At the same time, the formation of $\mathrm{PI}^{*}$ radical (3) and the interaction of this radical with $\mathrm{AgNO}_{3}$ (5) can further reduce silver ions to AgNPs. Further increase in PI concentration from 1 to $2 \mathrm{~g} / \mathrm{L}$ was accompanied by marginal decrease in the absorbance (nearly leveling off state is achieved). This could be due to the formation of the pinnacol derivative (I) (4) which, in its turn, leads to the inactivation of the photosynthesis of AgNPs.

The four sharp bands at $256 \mathrm{~nm}$ are representing the PI. As is clear (Figure 1), the intensity of the bands increases with increasing the concentration of PI from 0.5 to $2 \mathrm{~g} / \mathrm{L}$ reflecting a steady state of concentration of PI. The consumption of the $\mathrm{PI}$ in formation of $\mathrm{PI}^{\bullet}$ radical (3) or the pinnacol derivative (I) (4) is amended by the reformation of PI (5) according to its "life circle" shown in (1)-(5).

3.3. Effect of Silver Nitrate Concentration on the Synthesis of Silver Nanoparticles. To study the effect of increasing the concentrations of $\mathrm{AgNO}_{3}$ on the photosynthesis of AgNPs, different concentrations of $\mathrm{AgNO}_{3}$ (from 0.5 to $2 \mathrm{~g} / \mathrm{L}$ ) were used. Thus, photosynthesis was carried out using PI/UV system for one hour of UV irradiation at $30^{\circ} \mathrm{C}$ using CMS and PI concentrations of $50 \mathrm{~g} / \mathrm{L}$ and $1 \mathrm{~g} / \mathrm{L}$, respectively, and a $\mathrm{M}: \mathrm{L}$ ratio $1: 20$ at $\mathrm{pH}$ 7. The dependence of the absorbance of the colloidal solution of the synthesized AgNPs on the concentration of $\mathrm{AgNO}_{3}$ is shown in Figure 2. It shows three intensive bell shaped bands at $\mathrm{AgNO}_{3}$ concentration of $0.5 \mathrm{~g} / \mathrm{L}, 1 \mathrm{~g} / \mathrm{L}$,

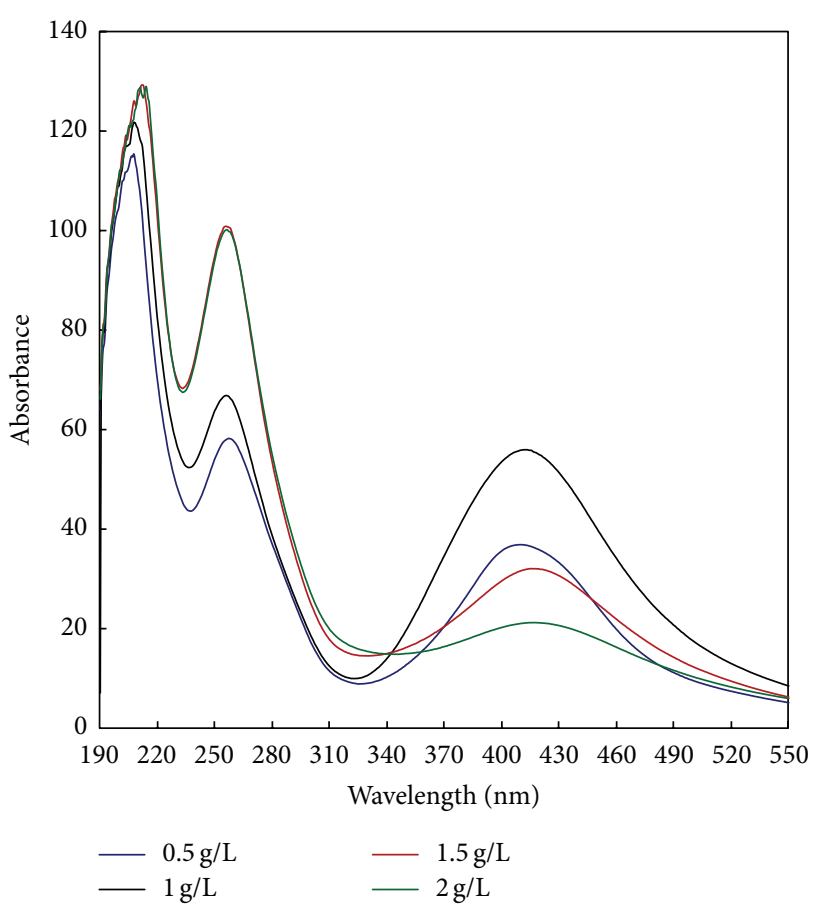

FIGURE 2: Effect of silver nitrate concentration on the synthesis of silver nanoparticles [CMS], $50 \mathrm{~g} / \mathrm{L} ;[\mathrm{PI}], 1 \mathrm{~g} / \mathrm{L} ;\left[\mathrm{AgNO}_{3}\right], 0.5-2 \mathrm{~g} / \mathrm{L}$; temp., $30^{\circ} \mathrm{C}$; time, 60 min.; $\mathrm{M}: \mathrm{L}$ ratio, $1: 20 ; \mathrm{pH}, 7$.

and $1.5 \mathrm{~g} / \mathrm{L}$. The fourth band representing $\mathrm{AgNO}_{3}$ concentration of $2 \mathrm{~g} / \mathrm{L}$ band is broad without the bell shape characteristics. The figure also shows that the absorbance of the colloidal solution increases by increasing the concentration of $\mathrm{AgNO}_{3}$ from 0.5 to $1 \mathrm{~g} / \mathrm{L}$ after which it decreases. It seems from the above findings that incorporation of PI into the PI/UV system in a concentration of $1 \mathrm{~g} / \mathrm{L}$ enhances the formation of $\mathrm{CMS}^{\circ}$ radical and $\mathrm{PI}^{*}(3)$ in an amount just enough for the conversion of the $\mathrm{Ag}^{+}$to $\mathrm{Ag}^{0}$ when the concentration of $\mathrm{AgNO}_{3}$ is in the range of $0.5-1 \mathrm{~g} / \mathrm{L}$. More reducing groups and more radicals are required to guarantee the conversion of all $\mathrm{Ag}^{+}$to $\mathrm{Ag}^{0}$ when $\mathrm{AgNO}_{3}$ concentration is to be increased to more than $1 \mathrm{~g} / \mathrm{L}$. This could be achieved by increasing both PI and $\mathrm{AgNO}_{3}$ concentration together in a parallel way. It is also likely that higher concentration of $\mathrm{AgNO}_{3}$ acts in favor of agglomeration of the silver particles rather than in the formation of capped silver nanoparticles in a colloidal solution [5].

3.4. Effect of Carboxymethyl Starch Concentration on the Synthesis of Silver Nanoparticles. Figure 3 shows the UVvis absorption spectra of the colloidal solution of AgNPs prepared using different concentrations of CMS (10-40 g/L). The data reveal that, regardless of the CMS concentration used, four similar bands are formed at the four concentrations of CMS with a symmetrical bell shape which is characteristic of the formation of AgNPs. It is clear also that there is a gradual decrease in the absorption intensity by increasing the CMS concentration from 10 to $40 \mathrm{~g} / \mathrm{L}$. It should be mentioned that the least amount of CMS in the reaction 


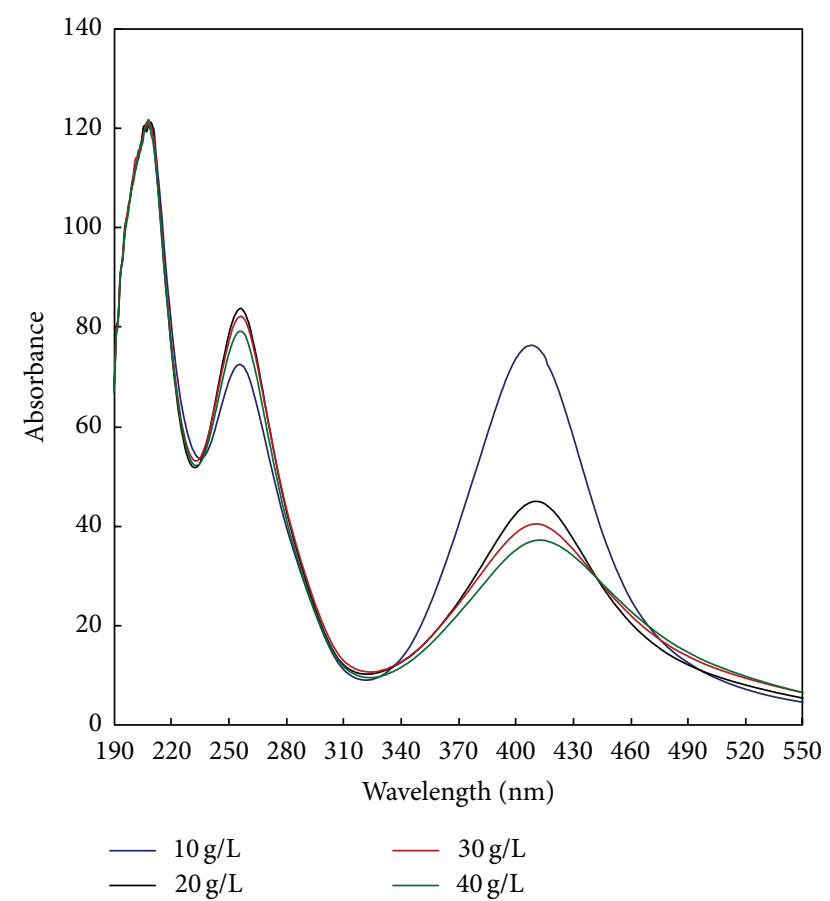

Figure 3: Effect of carboxymethyl starch concentration on the synthesis of silver nanoparticles [CMS], 10-40 g/L; [PI], $1 \mathrm{~g} / \mathrm{L}$; $\left[\mathrm{AgNO}_{3}\right], 1 \mathrm{~g} / \mathrm{L}$; temp., $30^{\circ} \mathrm{C}$; time, 60 min.; $\mathrm{M}: \mathrm{L}$ ratio, $1: 20 ; \mathrm{pH}, 7$.

medium $(10 \mathrm{~g} / \mathrm{L})$ together with $1 \mathrm{~g} / \mathrm{L}$ of PI is enough for full reduction of the $\mathrm{Ag}^{+}$to $\mathrm{Ag}^{0}$ and efficient for stabilization of the formed AgNPs. The decrease in the absorbance as a result of increasing the concentration of CMS could be ascribed to the very concentrated mixture which decreases the mobility of PI and $\mathrm{AgNO}_{3}$ and hinders the conversion of $\mathrm{Ag}^{+}$to $\mathrm{Ag}^{0}$.

3.5. Effect of Irradiation Time on the Synthesis of Silver Nanoparticles. The irradiation dose was controlled by controlling the irradiation time; this is also the time of the photosynthesis reaction. A study of the irradiation time took place using irradiation duration from 30 to $120 \mathrm{~min}$. The dependence of the absorbance of the colloidal solution of the synthesized AgNPs on the irradiation time is shown in Figure 4. Results in Figure 4 reveal that, regardless of irradiation time, four similar bands are formed at the four irradiation durations with a symmetrical bell shape which is characteristic of the formation of AgNPs. It can also be seen from the figure that increasing the irradiation time from 30 to $60 \mathrm{~min}$ is accompanied by an increase in the absorbance. It is also clear that absorbance values at $60-120 \mathrm{~min}$ are nearly the same showing a state of leveling off. The favorable effect of increasing the irradiation time on the synthesis of AgNPs is due to the formation of $\mathrm{PI}^{*}$ radicals which, in turn, produce the $\mathrm{CMS}^{\circ}$ radicals by $\mathrm{H}$-abstraction from CMS. The role of these radicals in the conversion of $\mathrm{Ag}^{+}$to $\mathrm{Ag}^{0}$ is clearly described in Section 3.2. Leveling off at 60-120 min means that no more conversion to silver nanoparticles will take place at the set of reaction conditions. It should be mentioned that

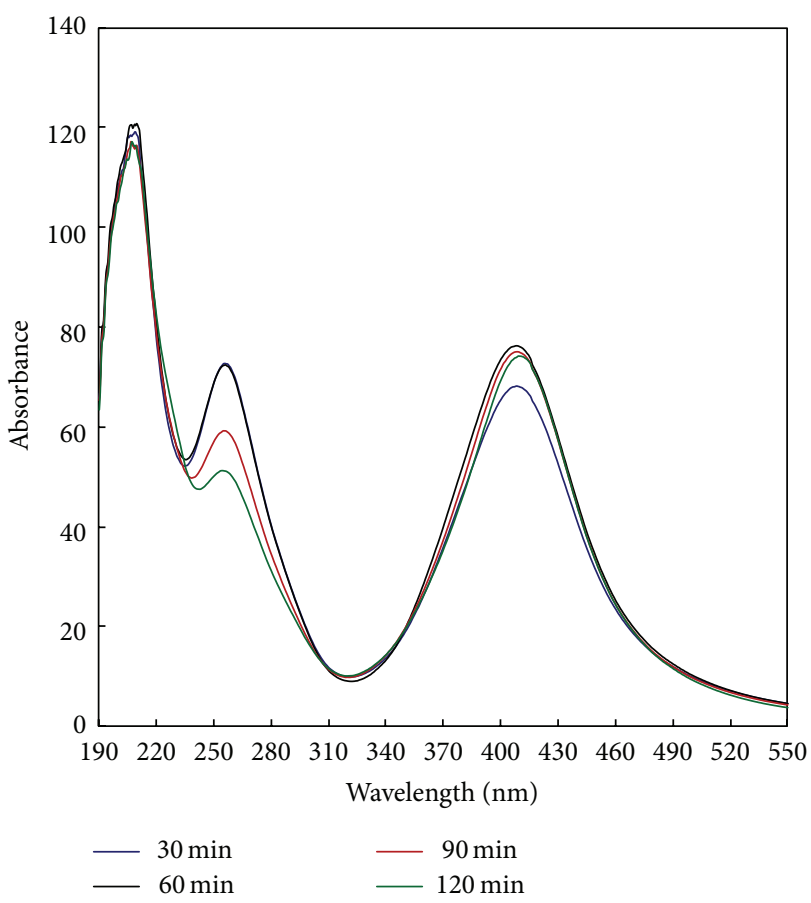

FIGURE 4: Effect of irradiation time on the synthesis of silver nanoparticles [CMS], $10 \mathrm{~g} / \mathrm{L} ;[\mathrm{PI}], 1 \mathrm{~g} / \mathrm{L} ;\left[\mathrm{AgNO}_{3}\right.$ ], $1 \mathrm{~g} / \mathrm{L}$; temp., $30^{\circ} \mathrm{C}$; time, 30-120 min.; $\mathrm{M}$ : L ratio, 1:20; $\mathrm{pH}, 7$.

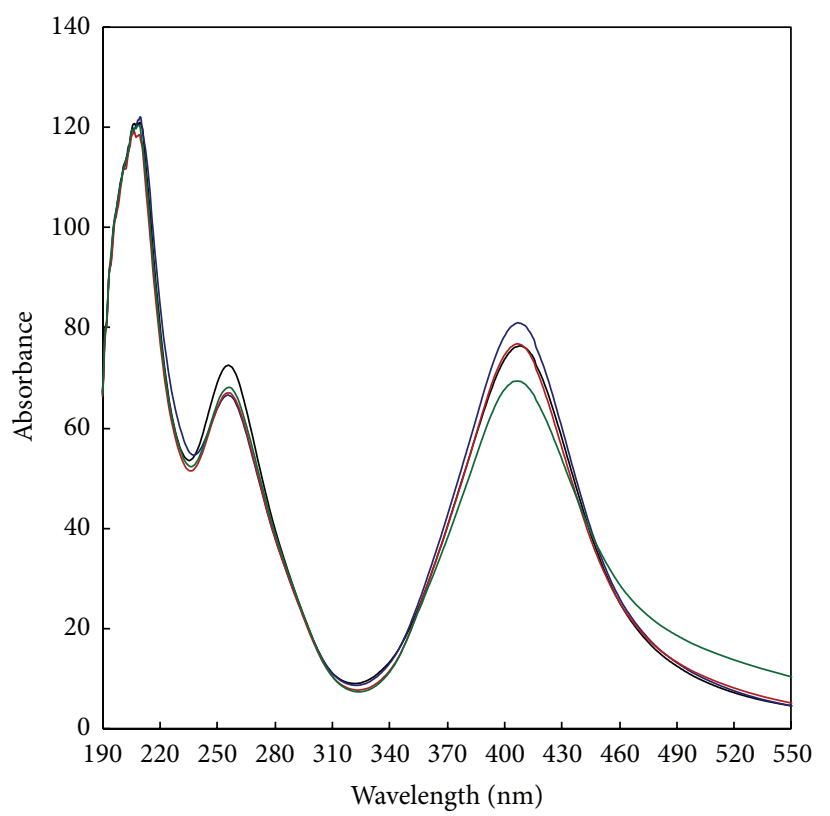

Temperature $\left({ }^{\circ} \mathrm{C}\right)$

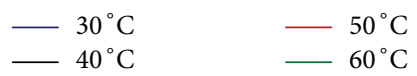

Figure 5: Effect of reaction temperature on the synthesis of silver nanoparticles [CMS], $10 \mathrm{~g} / \mathrm{L}$; [PI], $1 \mathrm{~g} / \mathrm{L}$; $\left[\mathrm{AgNO}_{3}\right.$ ], $1 \mathrm{~g} / \mathrm{L}$; temp., 30$60^{\circ} \mathrm{C}$; time, $60 \mathrm{~min}$.; $\mathrm{M}$ : $\mathrm{L}$ ratio, $1: 20 ; \mathrm{pH}, 7$. 


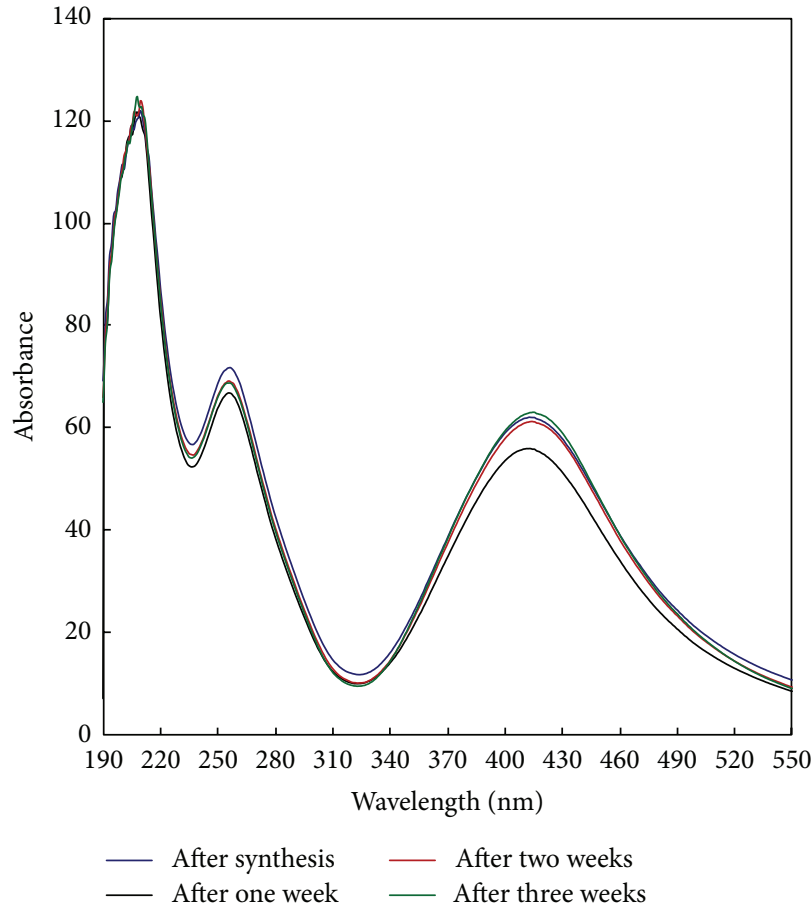

(a)

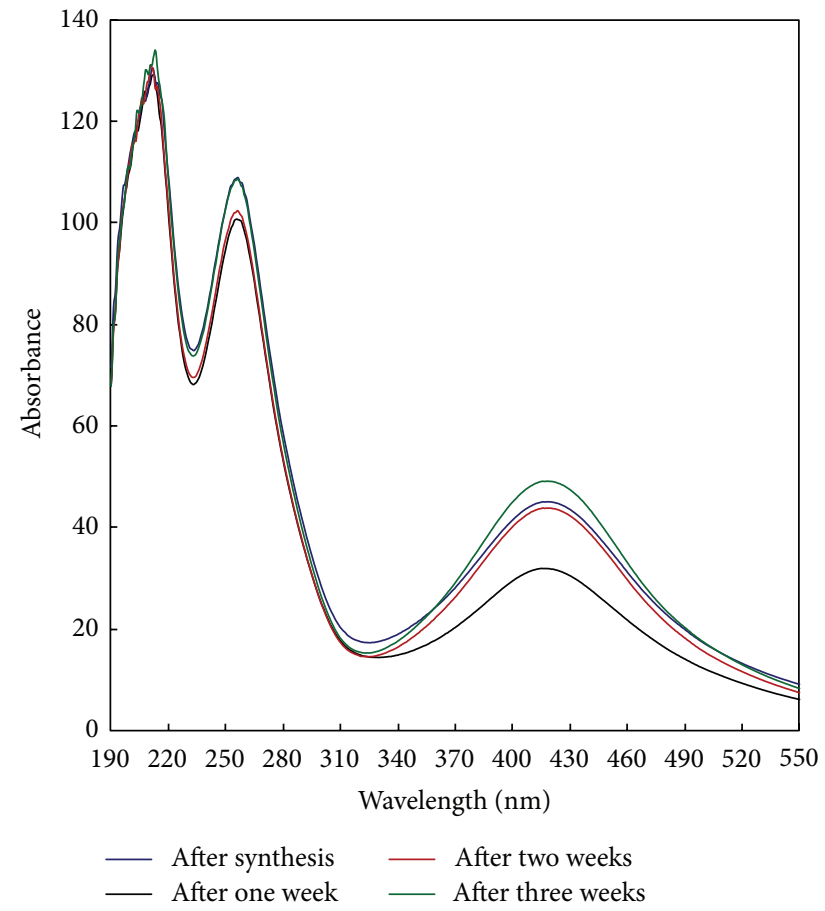

(b)

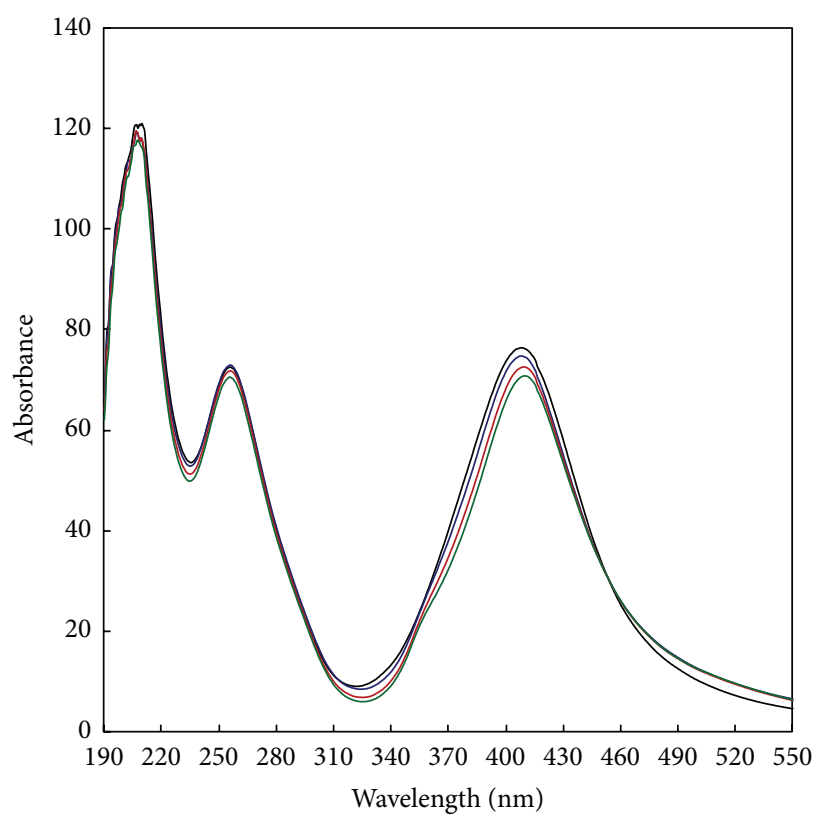

$\begin{array}{ll}- \text { After synthesis } & - \text { After two weeks } \\ - \text { After one week } & \quad \text { After three weeks }\end{array}$

(c)

FIGURE 6: Stability of silver nanoparticles synthesized at different conditions after one week, two weeks, and three weeks of storing. (a) [CMS], 50 g/L; [PI], 1 g/L; [ $\mathrm{AgNO}_{3}$ ], $1 \mathrm{~g} / \mathrm{L}$; temp., $30^{\circ} \mathrm{C}$; time, 60 min.; M: L ratio, $1: 20 ; \mathrm{pH}$, 7. (b) [CMS], 50 g/L; [PI], 1g/L; [AgNO $]$ ], $1.5 \mathrm{~g} / \mathrm{L} ;$ temp., $30^{\circ} \mathrm{C}$; time, $60 \mathrm{~min}$.; $\mathrm{M}: \mathrm{L}$ ratio, $1: 20 ; \mathrm{pH}$, 7. (c) [CMS], $10 \mathrm{~g} / \mathrm{L} ;[\mathrm{PI}], 1 \mathrm{~g} / \mathrm{L} ;\left[\mathrm{AgNO}_{3}\right], 1 \mathrm{~g} / \mathrm{L} ;$ temp., $30^{\circ} \mathrm{C} ;$ time, $60 \mathrm{~min}$.; $\mathrm{M}: \mathrm{L}$ ratio, $1: 20 ; \mathrm{pH}, 7$. 

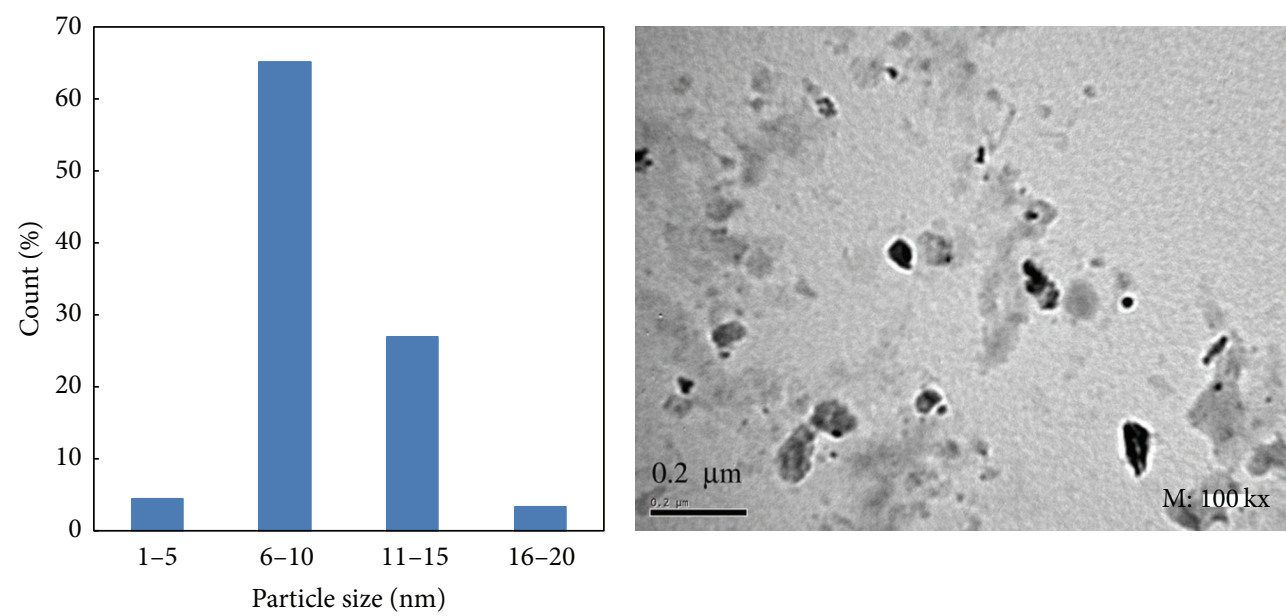

(a)
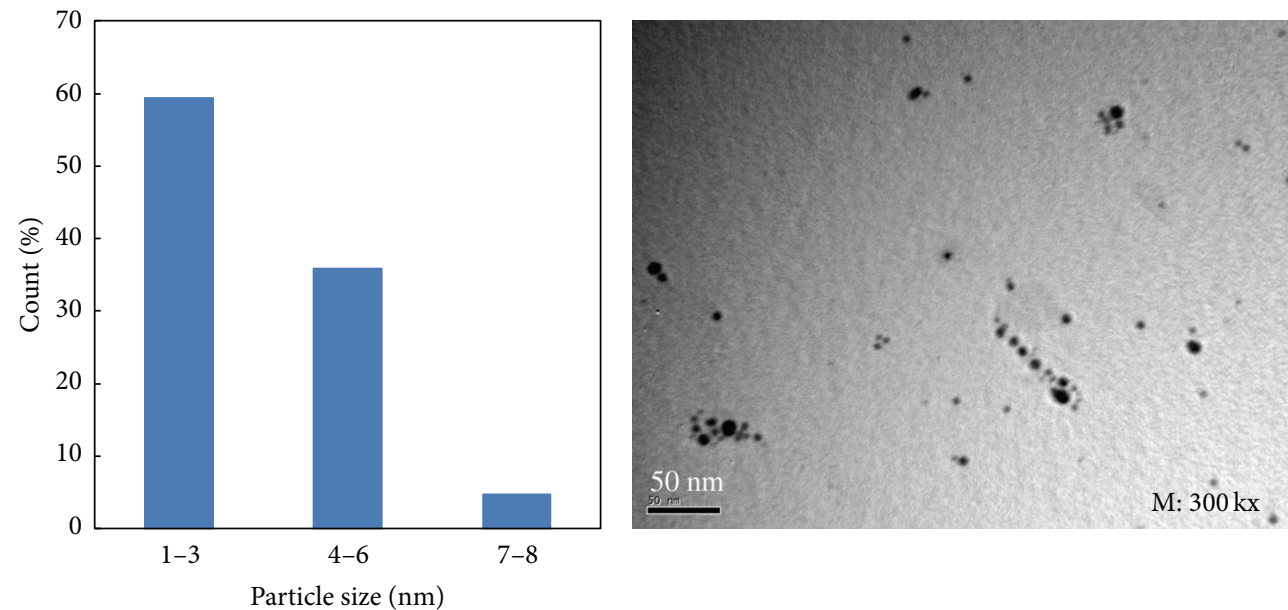

(b)
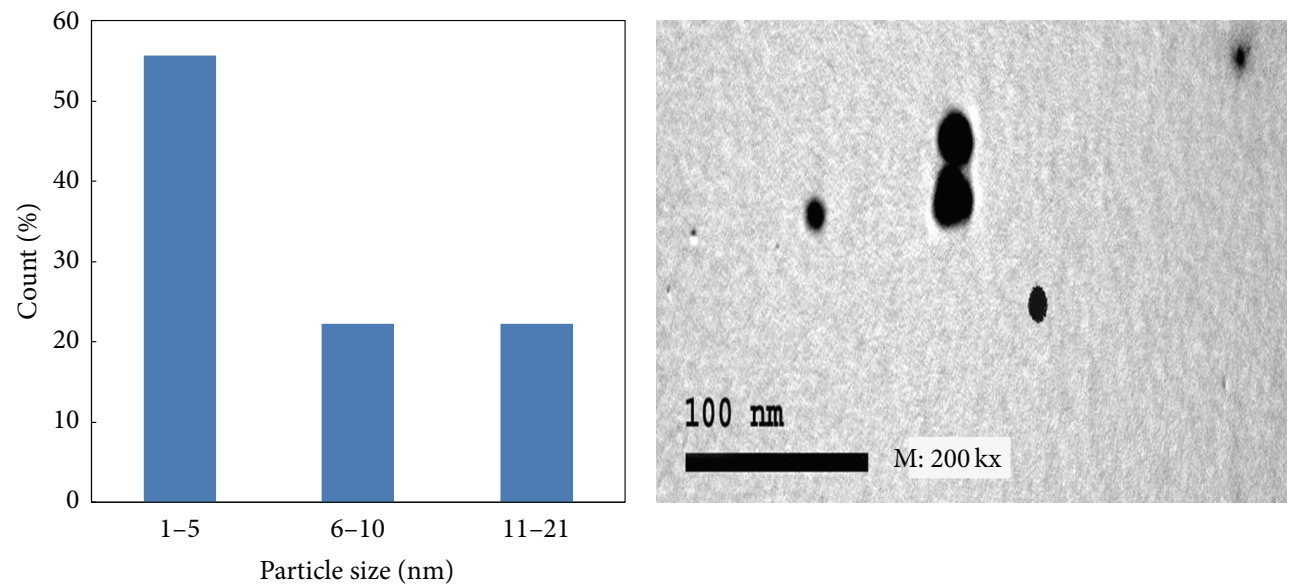

(c)

FIGURE 7: TEM micrograph and particle size distribution histogram of silver nanoparticles synthesized at different synthesis conditions. (a) [CMS], 50 g/L; [PI], $1 \mathrm{~g} / \mathrm{L} ;\left[\mathrm{AgNO}_{3}\right.$ ], $1 \mathrm{~g} / \mathrm{L}$; temp., 30 $\mathrm{C}$; time, 60 min.; $\mathrm{M}: \mathrm{L}$ ratio, $1: 20 ; \mathrm{pH}$, 7. (b) [CMS], $50 \mathrm{~g} / \mathrm{L} ;[\mathrm{PI}], 1 \mathrm{~g} / \mathrm{L} ;[\mathrm{AgNO}$ ] $] 1.5 \mathrm{~g} / \mathrm{L}$; temp., $30^{\circ} \mathrm{C}$; time, 60 min.; $\mathrm{M}: \mathrm{L}$ ratio, $1: 20 ; \mathrm{pH}$, 7. (c) [CMS], $10 \mathrm{~g} / \mathrm{L} ;[\mathrm{PI}], 1 \mathrm{~g} / \mathrm{L} ;\left[\mathrm{AgNO}_{3}\right], 1 \mathrm{~g} / \mathrm{L}$; temp., $30^{\circ} \mathrm{C}$; time, $60 \mathrm{~min}$.; $\mathrm{M}: \mathrm{L}$ ratio, $1: 20$; $\mathrm{pH}, 7$. 
$60 \mathrm{~min}$ of irradiation is enough for full reduction of the $\mathrm{Ag}^{+}$ to $\mathrm{Ag}^{0}$ nanoparticles.

3.6. Effect of Reaction Temperature on the Synthesis of Silver Nanoparticles. To study the effect of increasing the synthesis temperature on the photosynthesis of AgNPs, different temperatures (from 30 to $60^{\circ} \mathrm{C}$ ) were applied. The dependence of the absorbance of the colloidal solution of the synthesized AgNPs on the synthesis temperature is shown in Figure 5. Results in Figure 5 show that, regardless of photosynthesis temperature, similar bands are formed at all the photosynthesis temperatures under study with a symmetrical bell shape which is characteristic of the formation of AgNPs. The results obtained from Figure 5 also show that increasing the photosynthesis temperature from $30^{\circ} \mathrm{C}$ to $40^{\circ} \mathrm{C}$ is accompanied by an increase in the absorbance followed by a decrease in the absorbance by raising the temperature to $50-60^{\circ} \mathrm{C}$. Increasing the temperature favors the formation of $\mathrm{PI}^{\bullet}$ radicals which, in turn, produce the $\mathrm{CMS}^{-}$radicals by $\mathrm{H}$-abstraction from CMS. The role of these radicals in the conversion of $\mathrm{Ag}^{+}$ to $\mathrm{Ag}^{0}$ is clearly described in Section 3.2. Further increase in the reaction temperature $\left(50-60^{\circ} \mathrm{C}\right)$ favors the formation of the pinnacol derivative (I) according to (4). Consequently, the inactivation of the photosynthesis process is more pronounced.

3.7. Stability of Silver Nanoparticles Synthesized at Different Conditions after One Week, Two Weeks, and Three Weeks of Storing. Stability of photosynthesized AgNPs was tested by measuring the absorbance of the colloidal solution after synthesis, after one week, after two weeks, and after three weeks of storing. Figure 6 shows absorbance of the colloidal solution of samples synthesized at different conditions.

Figure 6 reveals that, regardless of storing duration and the set of the synthesis conditions, similar bands are formed for all samples with a symmetrical bell shape which is characteristic of the AgNPs. Figure 6(a) shows that the absorbance curves and the absorbance maxima after one, two, and three weeks of storing are identical with an even higher absorbance value than that after synthesis. These data reveal that up to one week of storing, the reaction was continued at room temperature until all silver ions are converted to AgNPs followed by stability of the colloidal solution after such a long period of storing. Figure 6(b) confirms the findings of Figure 6(a) that the lowest absorbance is related to the AgNPs of the freshly prepared sample and that storing even enhances the complete conversion of silver ions to AgNPs. In Figure 6(c), the case is different and the highest absorbance was related to the freshly prepared sample. Marginal decrease was noticed in the absorbance of samples stored for one, two, and three weeks, respectively. The decrease in the absorbance is very little and does not seem to affect the stability of the synthesized AgNPs. The only affecting parameter among samples $a, b$, and $c$ is the concentration of CMS. CMS concentration in samples $\mathrm{a}$ and $\mathrm{b}$ is $50 \mathrm{~g} / \mathrm{L}$ while it is $10 \mathrm{~g} / \mathrm{L}$ for sample c. This may explain the continuity of the synthesis reaction for samples "a" and "b" but not for sample "c" where the initiation step successfully generated the CMS ${ }^{\circ}$ radical which, in its turn, initiates an oxidation reaction resulting in the generation of new aldehydic end groups capable of reducing $\mathrm{Ag}^{+}$to $\mathrm{Ag}^{0}$. Although such a reaction is very slow and constitutes a minor route for $\mathrm{Ag}^{+}$reduction at such a set of reaction conditions (room temperature and $\mathrm{pH} 7$ ), the long storing time can finally lead to the formation of AgNPs.

3.8. TEM Micrograph and Particle Size Distribution Histogram of Silver Nanoparticles Synthesized at Different Synthesis Conditions. The same AgNPs samples used for the stability test were again used to establish the TEM micrograph and particle size distribution histogram. Figure 7 shows that, regardless of the reaction conditions, the three micrographs show that silver nanoparticles have round shape morphology and fine dispersion. Some aggregates were noticed in the micrograph of sample a. The mean particle sizes are between 1-20 nm, 1$8 \mathrm{~nm}$, and 1-21 nm for samples a, b, and c, respectively. Some irregularly shaped particles were found in Figure 7(a) which could be due to aggregations of some nanoparticles. The highest count $\%$ was found for AgNPs between 6-10, 1-3, and 1-5 for samples $\mathrm{a}, \mathrm{b}$, and $\mathrm{c}$, respectively.

\section{Conclusions}

4-(Trimethyl ammonium methyl) benzophenone chloride (a water soluble photoinitiator), PI/UV system, carboxymethyl starch (a water soluble ecofriendly polymer), and water (a solvent) were used to synthesis AgNPs using silver nitrate as a precursor. Thus, the chemicals and the process are green. The reduction of $\mathrm{Ag}^{+}$to $\mathrm{Ag}^{0}$ was successfully achieved via the generation of active $\mathrm{PI}^{*}$ and $\mathrm{CMS}^{*}$ free radicals. Optimal reaction conditions that yield the highest absorbance values were CMS, PI, and $\mathrm{AgNO}_{3}$ concentrations of $10 \mathrm{~g} / \mathrm{L}, 1 \mathrm{~g} / \mathrm{L}$, and $1 \mathrm{~g} / \mathrm{L}$, respectively, at $40^{\circ} \mathrm{C}$ for $60 \mathrm{~min}$ at $\mathrm{pH} 7$ using a $M: L$ ratio of $1: 20$. AgNPs so-obtained were stable in aqueous solution over a period of three weeks at room temperature $\left(\sim 25^{\circ} \mathrm{C}\right)$, and they have a round shape morphology. The sizes of synthesized AgNPs were found in the range of $1-21 \mathrm{~nm}$ and the highest counts $\%$ of these particles were for particles of $6-$ 10 and $1-3 \mathrm{~nm}$, respectively.

\section{Conflict of Interests}

The author declares that there is no conflict of interests regarding the publication of this paper.

\section{References}

[1] M. H. El-Rafie, A. A. Mohamed, T. I. Shaheen, and A. Hebeish, "Antimicrobial effect of silver nanoparticles produced by fungal process on cotton fabrics," Carbohydrate Polymers, vol. 80, no. 3, pp. 779-782, 2010.

[2] M. H. El-Rafie, A. A. Mohamed, T. I. Shaheen, and A. Hebeish, "Antimicrobial effect of silver nanoparticles produced by fungal process on cotton fabrics," Carbohydrate Polymers, vol. 80, no. 3, pp. 779-782, 2010.

[3] A. Hebeish, F. A. Abdel-Mohdy, M. M. G. Fouda et al., "Green synthesis of easy care and antimicrobial cotton fabrics," Carbohydrate Polymers, vol. 86, no. 4, pp. 1684-1691, 2011. 
[4] A. Hebeish, M. E. El-Naggar, M. M. G. Fouda, M. A. Ramadan, S. S. Al-Deyab, and M. H. El-Rafie, "Highly effective antibacterial textiles containing green synthesized silver nanoparticles," Carbohydrate Polymers, vol. 86, no. 2, pp. 936-940, 2011.

[5] A. Hebeish, A. El-Shafei, S. Sharaf, and S. Zaghloul, "Novel precursors for green synthesis and application of silver nanoparticles in the realm of cotton finishing," Carbohydrate Polymers, vol. 84, no. 1, pp. 605-613, 2011.

[6] G. . Schmid, "Syntheses and Characterizations: 3.3 Synthesis of Metal Nanoparticles," in Nanoparticles From Theory To Application, G. Schmid, Ed., pp. 214-239, Wiley-VCH Verlag GmbH \& Co. KGaA, Weinheim, Germany, 2010.

[7] P. Dallas, V. K. Sharma, and R. Zboril, "Silver polymeric nanocomposites as advanced antimicrobial agents: classification, synthetic paths, applications, and perspectives," Advances in Colloid and Interface Science, vol. 166, no. 1-2, pp. 119-135, 2011.

[8] N. Vigneshwaran, R. P. Nachane, R. H. Balasubramanya, and P. V. Varadarajan, "A novel one-pot 'green' synthesis of stable silver nanoparticles using soluble starch," Carbohydrate Research, vol. 341, no. 12, pp. 2012-2018, 2006.

[9] L. Balan and D. Burget, "Synthesis of metal/polymer nanocomposite by UV-radiation curing," European Polymer Journal, vol. 42, no. 12, pp. 3180-3189, 2006.

[10] L. Balan, M. Jin, J.-P. Malval, H. Chaumeil, A. Defoin, and L. Vidal, "Fabrication of silver nanoparticle-embedded polymer promoted by combined photochemical properties of a 2,7diaminofluorene derivative dye," Macromolecules, vol. 41, no. 23, pp. 9359-9365, 2008.

[11] L. Balan, J.-P. Malval, R. Schneider, D. Le Nouen, and D.-J. Lougnot, "In-situ fabrication of polyacrylate-silver nanocomposite through photoinduced tandem reactions involving eosin dye," Polymer, vol. 51, no. 6, pp. 1363-1369, 2010.

[12] L. Balan, R. Schneider, and D. J. Lougnot, "A new and convenient route to polyacrylate/silver nanocomposites by lightinduced cross-linking polymerization," Progress in Organic Coatings, vol. 62, no. 3, pp. 351-357, 2008.

[13] C. Decker, L. Keller, K. Zahouily, and S. Benfarhi, "Synthesis of nanocomposite polymers by UV-radiation curing," Polymer, vol. 46, no. 17, pp. 6640-6648, 2005.

[14] L. Keller, C. Decker, K. Zahouily, S. Benfarhi, J. M. Le Meins, and J. Miehe-Brendle, "Synthesis of polymer nanocomposites by UV-curing of organoclay-acrylic resins," Polymer, vol. 45, no. 22, pp. 7437-7447, 2004.

[15] Y. Yagci, O. Sahin, T. Ozturk, S. Marchi, S. Grassini, and M. Sangermano, "Synthesis of silver/epoxy nanocomposites by visible light sensitization using highly conjugated thiophene derivatives," Reactive and Functional Polymers, vol. 71, no. 8, pp. 857-862, 2011.

[16] Y. Yagci, M. Sangermano, and G. Rizza, "A visible light photochemical route to silver-epoxy nanocomposites by simultaneous polymerization-reduction approach," Polymer, vol. 49, no. 24, pp. 5195-5198, 2008.

[17] L. Balan, J. P. Malval, and D. J. Lougnot, "In situ photochemically assisted synthesis of silver nanoparticles in polymer matrixes," in Silver Nanoparticles, D. P. Perez, Ed., pp. 79-92, In-Tech, Croatia, 2010.

[18] J. Chen, J. Wang, X. Zhang, and Y. Jin, "Microwave-assisted green synthesis of silver nanoparticles by carboxymethyl cellulose sodium and silver nitrate," Materials Chemistry and Physics, vol. 108, no. 2-3, pp. 421-424, 2008.

[19] V. Djoković, R. Krsmanović, D. K. Božanić et al., "Adsorption of sulfur onto a surface of silver nanoparticles stabilized with sago starch biopolymer," Colloids and Surfaces B, vol. 73, no. 1, pp. 30-35, 2009.

[20] M. H. El-Rafie, M. E. El-Naggar, M. A. Ramadan, M. M. G. Fouda, S. S. Al-Deyab, and A. Hebeish, "Environmental synthesis of silver nanoparticles using hydroxypropyl starch and their characterization," Carbohydrate Polymers, vol. 86, no. 2, pp. 630-635, 2011.

[21] A. A. Hebeish, M. H. El-Rafie, F. A. Abdel-Mohdy, E. S. AbdelHalim, and H. E. Emam, "Carboxymethyl cellulose for green synthesis and stabilization of silver nanoparticles," Carbohydrate Polymers, vol. 82, no. 3, pp. 933-941, 2010.

[22] H. Huang, Q. Yuan, and X. Yang, "Preparation and characterization of metal-chitosan nanocomposites," Colloids and Surfaces $B$, vol. 39, no. 1-2, pp. 31-37, 2004.

[23] M. Z. Kassaee, A. Akhavan, N. Sheikh, and R. Beteshobabrud, " $\gamma$-Ray synthesis of starch-stabilized silver nanoparticles with antibacterial activities," Radiation Physics and Chemistry, vol. 77, no. 9, pp. 1074-1078, 2008.

[24] W. Liu, Z. Zhang, H. Liu, W. He, X. Ge, and M. Wang, "Silver nanorods using HEC as a template by $\gamma$-irradiation technique and absorption dose that changed their nanosize and morphology," Materials Letters, vol. 61, no. 8-9, pp. 1801-1804, 2007.

[25] P. Raveendran, J. Fu, and S. L. Wallen, "A simple and "green" method for the synthesis of $\mathrm{Au}, \mathrm{Ag}$, and $\mathrm{Au}-\mathrm{Ag}$ alloy nanoparticles," Green Chemistry, vol. 8, no. 1, pp. 34-38, 2006.

[26] V. K. Sharma, R. A. Yngard, and Y. Lin, "Silver nanoparticles: green synthesis and their antimicrobial activities," Advances in Colloid and Interface Science, vol. 145, no. 1-2, pp. 83-96, 2009.

[27] M. Valodkar, A. Bhadoria, J. Pohnerkar, M. Mohan, and S. Thakore, "Morphology and antibacterial activity of carbohydrate-stabilized silver nanoparticles," Carbohydrate Research, vol. 345, no. 12, pp. 1767-1773, 2010.

[28] S. Kaviya, J. Santhanalakshmi, and B. Viswanathan, "Biosynthesis of silver nano-flakes by Crossandra infundibuliformis leaf extract," Materials Letters, vol. 67, no. 1, pp. 64-66, 2012.

[29] Z. Sadowski, "Biosynthesis and application of silver and gold nanoparticles," in Silver Nanoparticles, D. P. Perez, Ed., pp. 257276, In Tech, 2010.

[30] A. Saxena, R. M. Tripathi, F. Zafar, and P. Singh, "Green synthesis of silver nanoparticles using aqueous solution of Ficus benghalensis leaf extract and characterization of their antibacterial activity," Materials Letters, vol. 67, no. 1, pp. 91-94, 2012.

[31] P. Vankar and D. Shukla, "Biosynthesis of silver nanoparticles using lemon leaves extract and its application for antimicrobial finish on fabric," Applied Nanoscience, vol. 2, no. 2, pp. 163-168, 2012.

[32] J. S. Bradley, G. Schmid, D. V. Talapin, E. V. Shevchenko, and H. Weller, "Syntheses and characterizations: 3.2 synthesis of metal nanoparticles," in Nanoparticles From Theory To Application, pp. 185-238, Wiley-VCH Verlag GmbH \& Co. KGaA, 2005.

[33] R. A. Bottom, J. T. Guthrie, and P. N. Green, "The influence of $\mathrm{H}$-donors on the photodecomposition of selected water-soluble photoinitiators," Polymer Photochemistry, vol. 6, no. 1, pp. 59-70, 1985.

[34] R. A. Bottom, J. T. Guthrie, and P. N. Green, "The photochemically induced grafting of 2-hydroxyethyl acrylate onto regenerated cellulose films from aqueous solutions," Polymer Photochemistry, vol. 6, no. 2, pp. 111-123, 1985.

[35] M. A. El-Sheikh, Synthesis of New Polymeric Materials Based on Water-Soluble Starch Composites [Ph.D. thesis], Faculty of Science Cairo University, Egypt, 1999. 
[36] M. A. El-Sheikh, "Photo grafting of acrylamide onto carboxymethyl starch I. Utilization of the product in easy care finishing of cotton fabric," in Proceedings of the 3rd Aachen-Dresden International Textile Conference, pp. 1-30, Aachen, 2009.

[37] M. A. El-Sheikh, M. A. Ramadan, and A. El-Shafie, "Photo oxidation of rice starch II. Using a water soluble photo initiator," Carbohydrate Polymers, vol. 78, no. 2, pp. 235-239, 2009.

[38] M. A. ElSheikh and J. T. Guthrie, "Graft copolymerization of acrylic acid onto carboxymethyl starch using UV-irradiation," in Proceedings of the 213th National Meeting, A. C. Society, Ed., vol. 213, ACS, San Francisco, Calif, USA, 1997.

[39] M. A. El-Sheikh, "Carboxymethylation of maize starch at mild conditions," Carbohydrate Polymers, vol. 79, no. 4, pp. 875-881, 2010.

[40] G. C. Daul, R. M. Reinhardt, and J. D. Reid, "Preparation of soluble yarns by the carboxymethylation of cotton," Textile Research Journal, vol. 23, no. 10, pp. 719-726, 1953.

[41] E. S. Abdel-Halim, M. H. El-Rafie, and S. S. Al-Deyab, "Polyacrylamide/guar gum graft copolymer for preparation of silver nanoparticles," Carbohydrate Polymers, vol. 85, no. 3, pp. 692697, 2011. 

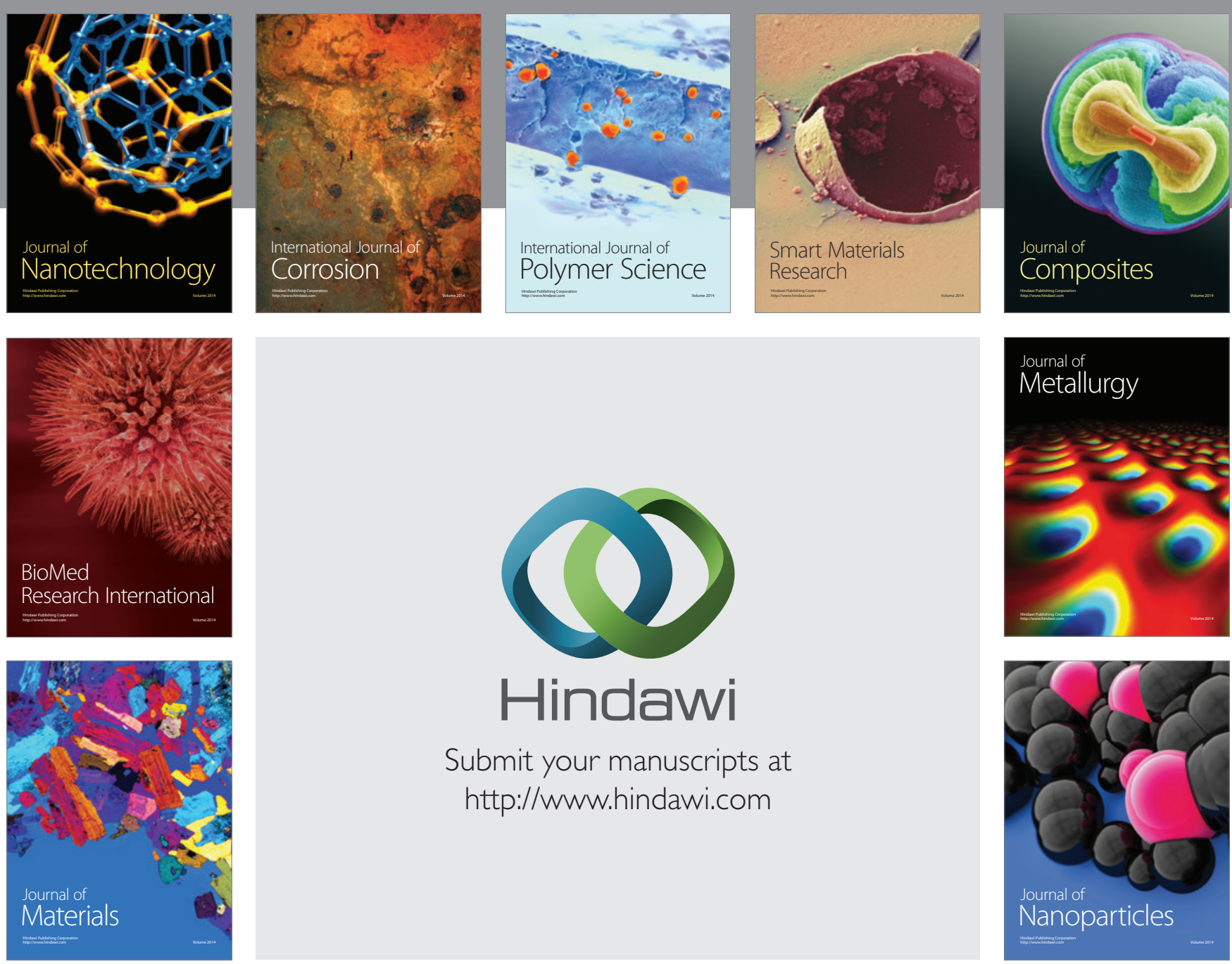

Submit your manuscripts at http://www.hindawi.com
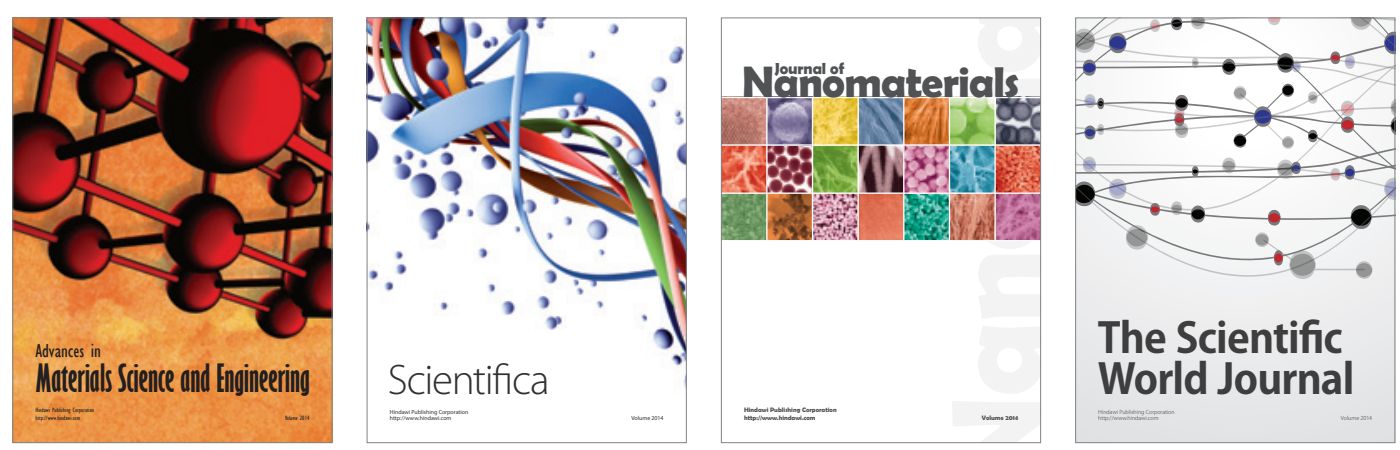

\section{The Scientific World Journal}
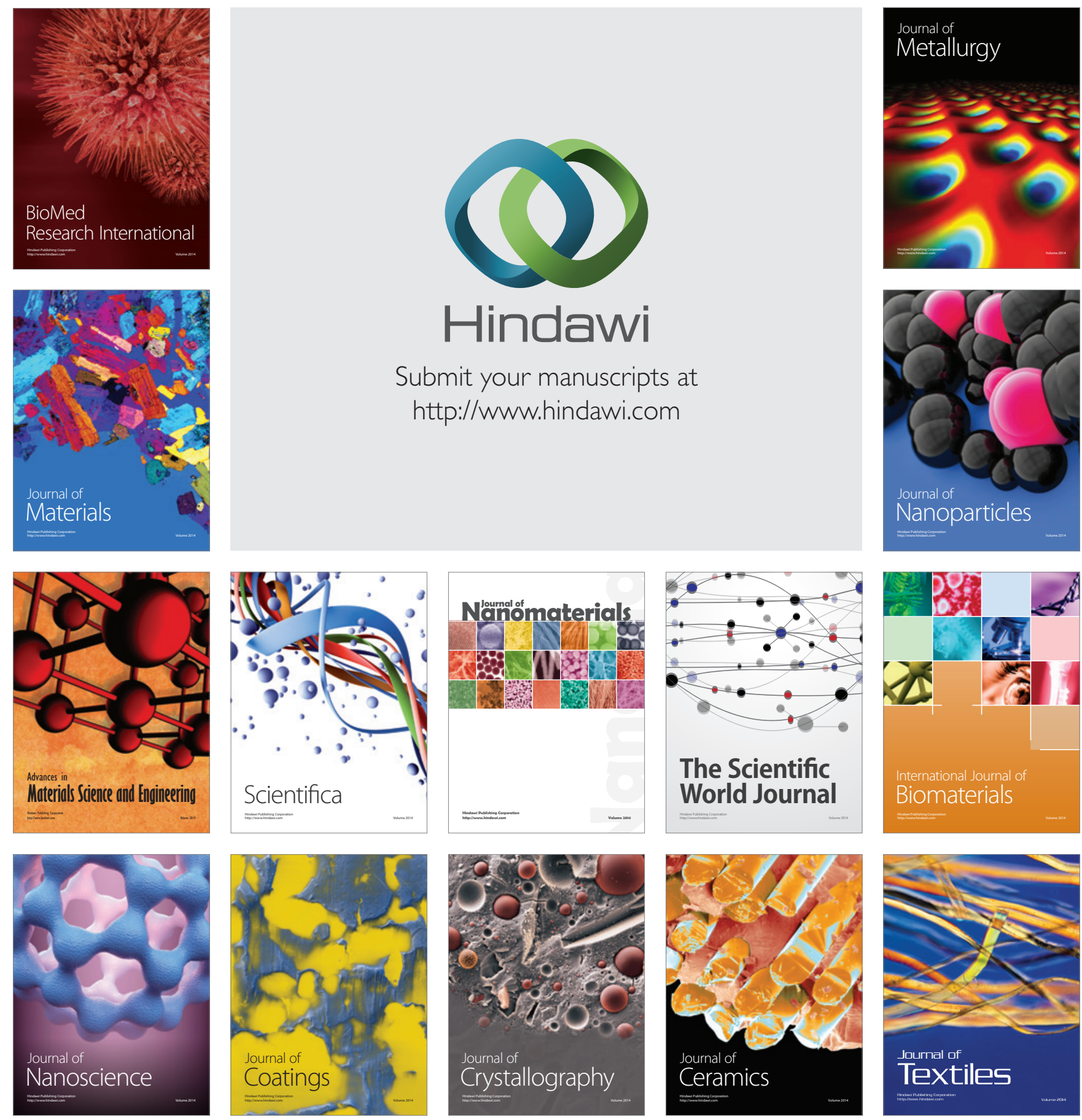